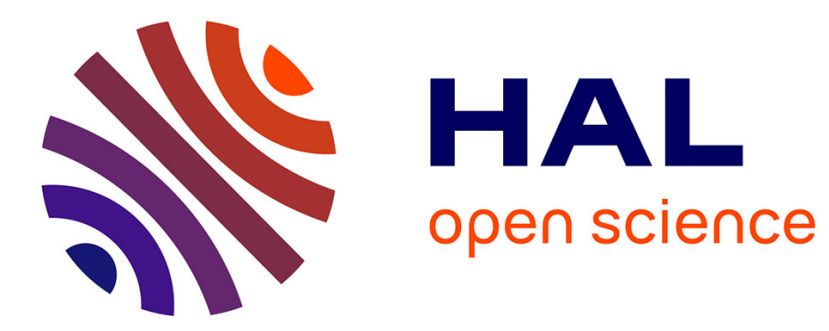

\title{
Skew-Product Decomposition of Planar Brownian Motion and Complementability
}

Jean Brossard, Michel Emery, Christophe Leuridan

\section{To cite this version:}

Jean Brossard, Michel Emery, Christophe Leuridan. Skew-Product Decomposition of Planar Brownian Motion and Complementability. Séminaire de Probabilités XLVI. Lecture Notes in Mathematics, vol 2123. Springer, pp.377-394, 2014, 10.1007/978-3-319-11970-0_15 . hal-02272534

\section{HAL Id: hal-02272534 https://hal.science/hal-02272534}

Submitted on 27 Aug 2019

HAL is a multi-disciplinary open access archive for the deposit and dissemination of scientific research documents, whether they are published or not. The documents may come from teaching and research institutions in France or abroad, or from public or private research centers.
L'archive ouverte pluridisciplinaire HAL, est destinée au dépôt et à la diffusion de documents scientifiques de niveau recherche, publiés ou non, émanant des établissements d'enseignement et de recherche français ou étrangers, des laboratoires publics ou privés. 


\title{
Skew-product decomposition \\ of planar Brownian motion and complementability
}

\author{
Jean Brossard, Michel Émery and Christophe Leuridan
}

August 27, 2019

Mathematical Classification : 60J65, 60H20.

Key words : Brownian filtrations, Planar Brownian motion, Skew-product decomposition, Complementability.

\begin{abstract}
Let $Z$ be a complex Brownian motion starting at 0 and $W$ the complex Brownian motion defined by

$$
W_{t}=\int_{0}^{t} \frac{\bar{Z}_{s}}{\left|Z_{s}\right|} \mathrm{d} Z_{s}
$$

The natural filtration $\mathcal{F}^{W}$ of $W$ is the filtration generated by $Z$ up to an arbitrary rotation. We show that given any two different matrices $Q_{1}$ and $Q_{2}$ in $O_{2}(\mathbf{R})$, there exists an $\mathcal{F}^{Z}$-previsible process $H$ taking values in $\left\{Q_{1}, Q_{2}\right\}$ such that the Brownian motion $\int H \cdot \mathrm{d} W$ generates the whole filtration $\mathcal{F}^{Z}$. As a consequence, for all $a$ and $b$ in $\mathbf{R}$ such that $a^{2}+b^{2}=1$, the Brownian motion $a \Re(W)+b \Im(W)$ is complementable in $\mathcal{F}^{Z}$.
\end{abstract}

\section{Introduction}

Brownian filtrations constitute a rich topic, where innocent-looking questions sometimes turn out to be quite tricky. How can one recognize if a given filtration is Brownian (that is, generated by some Brownian motion)? Few characterizations are known, and most of the time one has to exhibit a generating Brownian motion.

In 1980, Stroock and Yor [6] raised the following question.

(Q1): If a filtration $\mathcal{F}$ has the previsible representation property (PRP) w.r.t. some Brownian motion, is $\mathcal{F}$ necessarily Brownian?

Not until 15 years later was the matter settled, with two very different counterexamples provided by Dubins, Feldman, Smorodinsky and Tsirelson [3] and by Tsirelson [8]. The former shows the existence on Wiener space of a probability $Q$ equivalent to the Wiener measure $P$, such that no $Q$-BM generates the filtration (even though the filtration must have the PRP w.r.t. the $Q$-BM obtained as the Girsanov transform of $P$ ). And the latter counter-example asserts that the Walsh $\mathrm{BM}$ on three or more branches generates a non-Brownian filtration.

In view of the new light shed by these examples, an updated version of Stroock and Yor's original question can be asked. 
(Q2): If a filtration $\mathcal{F}$ is immersed ${ }^{1}$ in some (possibly infinite-dimensional)

Brownian filtration and has the PRP w.r.t. some $\mathcal{F}$-Brownian motion, is $\mathcal{F}$ necessarily Brownian?

The general case remains elusive, although a positive answer is obtained in [4] in the particular case of filtrations which are Brownian on every interval $[\varepsilon, \infty[$ with $\varepsilon>0$; under this very strong additional hypothesis, Brownianness or non Brownianness is a germ property at time $0+$. Natural examples of such filtrations are provided by quotienting the filtration of a $d$-dimensional Brownian motion by some subgroup of the orthogonal group. These filtrations are already proved to be Brownian by Malric in [5], who explicitly constructs Brownian motions generating them.

(Q2) draws attention to the various ways a Brownian filtration can be immersed in another Brownian filtration. For instance, given $Z$ a $d$-dimensional BM and given $k \in \llbracket 1, d-1 \rrbracket$, for each $k$-dimensional linear subspace $\mathrm{S}$ of $\mathbf{R}^{d}$ the orthogonal projection of $Z$ on $S$ is a BM whose filtration is immersed in the filtration $Z$ of $Z$, and all these immersions are clearly isomorphic to each other ${ }^{2}$. Moreover, given a $k$-dimensional $z$-BM $B$, its filtration is immersed in $z$, and this immersion is isomorphic to all previous ones if and only if there exists a $(d-k)$-dimensional z$\mathrm{BM} B^{\prime}$ independent of $B$ such that the $d$-dimensional $z$-BM $\left(B, B^{\prime}\right)$ generates the full filtration $z$. This property was introduced in [2] and called complementability; it is the simplest way a $k$-dimensional Brownian filtration can be immersed in a $d$-dimensional one.

Another property, maximality ${ }^{3}$, is defined in [2] and shown there to be necessary for complementability. This leads to the question of the sufficiency.

\section{(Q3): Conversely, does maximality imply complementability?}

Even in the simplest case, when $k=1$ and $d=2$, question (Q3) is still open. Although there is no direct mathematical relation between (Q2) and (Q3), they turn out to be similar, at two levels. First, each of them asks if some BM can be constructed, so as to generate (alone for (Q2), or together with $B$ for (Q3)) a given filtration. Second, in all instances so far that such a BM has successfully been constructed, the methods are similar, be it for (Q2) or (Q3); they rely on coupling arguments. It thus appears that studying (Q3) might indirectly contribute to progress on (Q2). At this stage, both (Q2) and (Q3) seem difficult: we have in view no strategy of proof, nor any candidate for a counter-example; but one of (Q2) and (Q3) may eventually turn out to be less difficult than the other.

The present work describes a family of real BM shown to be complementable in the filtration of a complex BM (Corollary); although these real BM are very simply defined, our proof of their complementability (by explicit construction of a complement) is rather involved.

From now on, we fix a complex Brownian motion $Z=X+\mathrm{i} Y$ starting at 0 . Almost surely, the Bessel process $R=|Z|$ never returns to 0 . We shall focus on the independent Brownian motions $\mathrm{U}$ and $\mathrm{V}$ given by

$$
U_{t}=\int_{0}^{t} \frac{X_{s} \mathrm{~d} X_{s}+Y_{s} \mathrm{~d} Y_{s}}{R_{s}} \quad \text { and } \quad V_{t}=\int_{0}^{t} \frac{X_{s} \mathrm{~d} Y_{s}-Y_{s} \mathrm{~d} X_{s}}{R_{s}} .
$$

The complex Brownian motion $W=U+\mathrm{i} V=\int(\bar{Z} / R) \mathrm{d} Z$ is known to generate the quotient filtration generated by $Z$ up to an arbitrary rotation.

Actually, $W$ is not the generating Brownian motion constructed by Malric in [5]. But among all Brownian motions generating the quotient filtration of $Z$ modulo

\footnotetext{
${ }^{1}$ A filtration $\mathcal{F}$ is said to be immersed in a filtration $\mathcal{G}$ when every $\mathcal{F}$-martingale is a $\mathcal{G}$-martingale.

${ }^{2}$ Given four filtrations $\mathcal{F}, \mathcal{G}, \mathcal{F}^{\prime}$ and $\mathcal{G}^{\prime}$ with $\mathcal{F}$ immersed in $\mathcal{G}$ and $\mathcal{F}^{\prime}$ immersed in $\mathcal{G}^{\prime}$, the immersion of $\mathcal{F}^{\prime}$ in $\mathcal{G}^{\prime}$ is isomorphic to the immersion of $\mathcal{F}$ in $\mathcal{G}$ if $\mathcal{G}$ and $\mathcal{G}^{\prime}$ are in correspondence by some isomorphism which maps $\mathcal{F}$ onto $\mathcal{F}^{\prime}$.

${ }^{3} \mathrm{~A} k$-dimensional z-BM $B$ is called maximal if no other $k$-dimensional z-BM generates a strictly bigger filtration than $B$.
} 
$\mathrm{SO}_{2}$, the complex Brownian motion $W=U+\mathrm{i} V$ is somehow the most natural one, since its real part $U$ governs the Bessel process $R$ whereas its imaginary part $V$ governs the increments of the angular part of $Z$. These facts are due to Stroock and Yor [7] and recalled in Proposition 1.

The complementability of $U$ is proved in [2] by exhibiting an independent complement. Is $V$ complementable too? In [1], $V$ is shown to be maximal, and its complementability is announced, without proof, at the end of the introduction. A proof will be given in the present paper; we shall actually establish a more general result as follows.

Let $Q_{1}$ and $Q_{2}$ be any two different matrices in $O_{2}(\mathbf{R})$. There exists an $\mathcal{F}^{Z}$-previsible process $H$ taking values in $\left\{Q_{1}, Q_{2}\right\}$ such that the Brownian motion $\int H \cdot \mathrm{d} W$ generates the whole filtration $\mathcal{F}^{Z}$.

Here, for every $Q \in \mathrm{O}_{2}(\mathbf{R})$ and $z \in \mathbf{C}$, we denote by $Q \cdot z$ the complex number provided by the usual action of the $2 \times 2$ real matrices on $\mathbf{C}$ identified with $\mathbf{R}^{2}$. More precisely, set

$$
Q=\left(\begin{array}{cc}
a & -\sigma b \\
b & \sigma a
\end{array}\right)
$$

with $a$ and $b$ in $\mathbf{R}, a^{2}+b^{2}=1, \sigma \in\{-1,1\}$, and put $c=a+\mathrm{i} b$. Then $Q \cdot z=c z$ or $Q \cdot z=c \bar{z}$ according to $\sigma$ being equal to 1 or -1 .

Observe that for each $Q \in O_{2}(\mathbf{R})$, the Brownian motion $\int(Q H) \cdot \mathrm{d} W=\int Q$. $\mathrm{d}\left(\int H \cdot \mathrm{d} W\right)$ generates the same filtration as $\int H \cdot \mathrm{d} W$. Hence, assuming that $Q_{2}=I_{2}$ in the proof of the theorem is no restriction. Moreover, Theorem is completely contained in its two corollaries below.

For every complex number $c \neq 1$ with modulus 1 , there exists an $\mathcal{F}^{Z}$-previsible process $\eta$ taking values in $\{1, c\}$ such that the Brownian motion $\int \eta \mathrm{d} W$ generates the whole filtration $\mathcal{F}^{Z}$.

For all $a$ and $b$ in $\mathbf{R}$ such that $a^{2}+b^{2}=1$, the Brownian motion $a U+b V$ is complementable in $\mathcal{F}^{Z}$.

Corollary directly follows from Theorem applied to the matrices $Q_{1}$ and $Q_{2}$ such that $Q_{1} \cdot z=z$ and $Q_{2} \cdot z=c z$. Similarly, Corollary follows from Theorem applied to the matrices

$$
Q_{1}=\left(\begin{array}{cc}
a & b \\
b & -a
\end{array}\right) \quad \text { and } \quad Q_{2}=\left(\begin{array}{cc}
a & b \\
-b & a
\end{array}\right) .
$$

Indeed, since $\Re\left(Q_{1} \cdot z\right)=\Re\left(Q_{2} \cdot z\right)=a \Re(z)+b \Im(z)$, the complex Brownian motion $\int H \cdot \mathrm{d} W$ provided by Theorem has real part $\Re\left(\int H \cdot \mathrm{d} W\right)=a U+b V$; so $a U+b V$ is complemented by the imaginary part $\Im\left(\int H \cdot \mathrm{d} W\right)$.

Note that the complementability of $U$, already proved in [2], corresponds to the choice $a=1$ and $b=0$ in formulas (2). Thus, what remains to be proved is Theorem when $Q_{2}=I_{2}$ and $Q_{1}=Q$ given by formula $(1)$ with $(a, b) \neq(1,0)$ and $\sigma \in\{-1,1\}$.

Actually, the proof given below for the case $(a, b) \neq(1,0)$ does not work any longer when $(a, b)=(1,0)$ and $\sigma=-1$ and must then be modified. We will explain why in subsection 2.4. Observe that the case when $(a, b)=(1,0)$ and $\sigma=1$ is not to be considered since it corresponds to $Q=I_{2}$.

The first section provides preliminary results whereas the second section is devoted to the proof.

\section{Notations and tools}

In the sequel, we fix a complex-valued Brownian motion $Z=X+\mathrm{i} Y$ started from 0 , and $U, V, W$ and $R$ are defined as above. The filtration $\mathcal{F}^{Z}$ generated by $Z$ will be the ambient filtration: unless otherwise specified, martingales, Brownian motions, stopping times are always relative to $\mathcal{F}^{Z}$. 
We fix an orthogonal matrix

$$
Q=\left(\begin{array}{cc}
a & -\sigma b \\
b & \sigma a
\end{array}\right)
$$

with $a$ and $b$ in $\mathbf{R}, a^{2}+b^{2}=1, \sigma \in\{-1,1\}$ and $a \neq 1$. Our aim is to construct a previsible process $H$ with values in $\left\{I_{2}, Q\right\}$ such that the complex Brownian motion $\widehat{W}=\int H \cdot \mathrm{d} W$ generates $\mathcal{F}^{Z}$.

Before introducing the tools for the construction of $H$, we recall some well-known facts (see Proposition 3.1 and Theorem 3.4 of Stroock-Yor [7]).

(Classical properties of $U$ and $V$.)

- The process $R$ is the unique and strong solution of the stochastic differential equation

$$
\mathrm{d} R_{s}=\mathrm{d} U_{s}+\frac{\mathrm{d} s}{2 R_{s}} ; \quad R_{0}=0 .
$$

In particular, the processes $R$ and $U$ generate the same filtration.

- For $t \geq s>0$,

$$
\frac{Z_{t}}{R_{t}}=\frac{Z_{s}}{R_{s}} \exp \left(\mathrm{i} \int_{s}^{t} \frac{\mathrm{d} V_{r}}{R_{r}}\right) .
$$

- Hence, given $t \geq s>0$, the value $Z_{t}$ can be recovered from $Z_{s}$ and from the increments of $W$ on the time-interval $[s, t]$.

- For each $t>0$, one has $\mathcal{F}_{t}^{Z}=\mathcal{F}_{t}^{W} \vee \sigma\left(Z_{t} / R_{t}\right)$, and the r.v. $Z_{t} / R_{t}$ is independent of $W$, with uniform law on the unit circle.

The fourth point of Proposition 1 describes the information missing in $W$ to recover $Z$, whereas the third point shows that the loss of information occurs only at time $0+$. This is a key fact in the proof of the next lemma.

Let $\left(C_{t}\right)_{0 \leq t \leq 1}$ be a complex $\mathcal{F}^{Z}$-Brownian motion such that the r.v. $Z_{1}$ is measurable in the $\sigma$-field $\mathcal{F}_{1}^{C}$. For $t>1$ set $C_{t}=C_{1}+W_{t}-W_{1}$. Then $\left(C_{t}\right)_{t \geq 0}$ is a Brownian motion which generates the whole filtration $\mathcal{F}^{Z}$.

By the third point of Proposition 1, since $C$ has the same increments as $W$ after time 1 , it suffices to show that $C$ generates on the time-interval $[0,1]$ the same filtration as $Z$. We only need to check that for every $t \in[0,1], Z_{t}$ is $\mathcal{F}_{t}^{C}$-measurable. Consider the $\mathcal{F}^{C}$-martingale $M$ given by $M_{t}=\mathbf{E}\left[Z_{1} \mid \mathcal{F}_{t}^{C}\right]$, whose final value $M_{1}$ is equal to $Z_{1}$ by our measurability hypothesis. As $C$ is an $\mathcal{F}^{Z}$-Brownian motion, every $\mathcal{F}^{C}$-martingale is an $\mathcal{F}^{Z}$-martingale (by the previsible representation property); so $M$ is on $[0,1]$ the $\mathcal{F}^{Z}$-martingale $\left(\mathbf{E}\left[Z_{1} \mid \mathcal{F}_{s}^{Z}\right]\right)_{s \in[0,1]}$, namely, $M=Z$ on $[0,1]$. Thus $Z_{t}$ is $\mathcal{F}_{t}^{C}$-measurable for $t \in[0,1]$.

Given a previsible process $\left(H_{t}\right)_{0<t \leq 1}$ with values in $\left\{I_{2}, Q\right\}$, one may apply Lemma 1 to the Brownian motion $C=\widehat{W}=\int H \cdot \mathrm{d} W$. To prove Theorem, it is thus sufficient to construct a previsible process $\left(H_{t}\right)_{0<t \leq 1}$ with values in $\left\{I_{2}, Q\right\}$ such that the r.v. $Z_{1}$ can be recovered from the Brownian motion $\left(\widehat{W}_{t}\right)_{0 \leq t \leq 1}$.

\subsection{Solution of a SDE governed by a complex Brownian mo- tion $C$}

Let $C=A+\mathrm{i} B$ be any complex $\mathcal{F}^{Z}$-Brownian motion. For $t \geq s>0$ and $\zeta$ any r.v. measurable in $\mathcal{F}_{s}^{Z}$ and valued in $\mathbf{C}^{*}$, we denote by $\operatorname{Sol}(C, s, t, \zeta)$ the value at time $t$ of the solution on the time interval $[s,+\infty$ [ of the stochastic differential equation

$$
\mathrm{d} Z_{t}^{\prime}=\frac{Z_{t}^{\prime}}{\left|Z_{t}^{\prime}\right|} \mathrm{d} C_{t} \quad \text { with initial condition } Z_{s}^{\prime}=\zeta .
$$

A priori, the solution to this SDE is only defined on some interval $[s, \gamma[$, where $\gamma$ is the hitting time of 0 by $Z^{\prime}$. But setting $\mathrm{d} Z^{\prime}=\mathrm{d} C$ on $\left[\gamma, \infty\left[\right.\right.$, one has $\mathrm{d} Z^{\prime}=H \mathrm{~d} C$ 
with $H$ previsible and $|H|=1$; so $Z^{\prime}$ is a complex BM, and $\gamma$, the hitting time of 0 by a complex BM, must therefore be a.s. infinite. This shows that the above equation was well posed, with solution $Z^{\prime}$ some complex BM started at time $s$ from the value $\zeta$. We have obtained the following statement.

The process $(\operatorname{Sol}(C, s, t, \zeta))_{t>s}$ is a complex Brownian motion starting from $\zeta$. Moreover, the random variable $\operatorname{Sol}(C, s, t, \zeta)$ depends only on $\zeta$ and on the increments of $C$ on the time interval $[s, t]$.

According to Proposition 1, the process $Z_{t}^{\prime}=\operatorname{Sol}(C, s, t, z)$ can be obtained by first solving on the time interval $[s,+\infty[$ the stochastic differential equation

$$
\mathrm{d} R_{t}^{\prime}=\mathrm{d} A_{t}+\frac{\mathrm{d} t}{2 R_{t}^{\prime}} \text { with initial condition } R_{s}^{\prime}=|\zeta|,
$$

and by then setting, for every $t \geq s$

$$
\frac{Z_{t}^{\prime}}{R_{t}^{\prime}}=\frac{\zeta}{|\zeta|} \exp \left(\mathrm{i} \int_{s}^{t} \frac{\mathrm{d} B_{r}}{R_{r}^{\prime}}\right)
$$

Formulas (3) and (4) show that two solutions associated to two initial conditions which have the same modulus evolve parallelly. This statement is made formal in the next lemma, which will repeatedly be used.

(Parallel evolution.) Given $s>0$, let $C=A+\mathrm{i} B$ be any complex $\mathcal{F}^{Z}$ Brownian motion and $\zeta^{\prime}$ and $\zeta^{\prime \prime}$ two $\mathcal{F}_{s}^{Z}$-measurable r.v. valued in $\mathbf{C}^{*}$. Then, on the event $\left[\left|\zeta^{\prime}\right|=\left|\zeta^{\prime \prime}\right|\right]$, one almost surely has

$$
\text { for all } t \geq s, \quad \frac{\operatorname{Sol}\left(C, s, t, \zeta^{\prime \prime}\right)}{\operatorname{Sol}\left(C, s, t, \zeta^{\prime}\right)}=\frac{\zeta^{\prime \prime}}{\zeta^{\prime}} .
$$

\subsection{Metrics on $\mathrm{C}^{*}$ and on $\mathrm{C}^{*}$-valued random variables}

Lemma 1.1 suggests that it is relevant to introduce the distance $d$ defined on the set $\mathbf{C}^{*}$ by

$$
\begin{array}{ll}
d\left(z, z^{\prime}\right)=2 \pi & \text { if }|z| \neq\left|z^{\prime}\right| \\
d\left(z, z^{\prime}\right)=\left|\arg \left(z^{\prime} / z\right)\right| & \text { if }|z|=\left|z^{\prime}\right|,
\end{array}
$$

where $\arg \left(z^{\prime} / z\right)$ is chosen in $[-\pi, \pi]$. Notice that $d\left(z, z^{\prime}\right) \leq \pi \Leftrightarrow|z|=\left|z^{\prime}\right|$. With this notation, the next result follows immediately from Lemma 1.1.

Let $s, C, \zeta^{\prime}$ and $\zeta^{\prime \prime}$ be as in Lemma 1.1. Almost surely, one has for all $t \geq s$

$$
d\left(\operatorname{Sol}\left(C, s, t, \zeta^{\prime}\right), \operatorname{Sol}\left(C, s, t, \zeta^{\prime \prime}\right)\right) \leq d\left(\zeta^{\prime}, \zeta^{\prime \prime}\right) .
$$

On the event $\left[\left|\zeta^{\prime}\right| \neq\left|\zeta^{\prime \prime}\right|\right]$ the right-hand side is $2 \pi$, the maximal value of $d$; on $\left[\left|\zeta^{\prime}\right|=\left|\zeta^{\prime \prime}\right|\right]$, Lemma 1.1 says that $d\left(\operatorname{Sol}\left(C, s, t, \zeta^{\prime}\right), \operatorname{Sol}\left(C, s, t, \zeta^{\prime \prime}\right)\right)$ remains constant in time.

It is not difficult to see that equality always holds in the conclusion of Corollary 1.2; but except in subsection 2.4 where the case that $(a, b)=(1,0)$ is discussed, we shall only need the majoration.

We come back to properties of $d$. For $\varepsilon<2 \pi$, the relation $d\left(z, z^{\prime}\right) \leq \varepsilon$ implies $\left|z-z^{\prime}\right| \leq \varepsilon|z|$; consequently, the $d$-topology is finer than the usual topology.

We shall also use the corresponding distance on random variables: since the metric $d$ is bounded, the formula $D\left(\zeta^{\prime}, \zeta^{\prime \prime}\right)=\mathbf{E}\left[d\left(\zeta^{\prime}, \zeta^{\prime \prime}\right)\right]$ defines a distance on the set of all random variables valued in $\mathbf{C}^{*}$ and defined up to almost sure equality. The topology associated to $D$ is nothing but the topology of convergence in probability for the metric $d$, and the comparison with the usual topology easily extends to random variables. 
Let $\left(\zeta_{n}\right)_{n>0}$ and $\zeta$ be random variables valued in $\mathbf{C}^{*}$, defined on $(\Omega, \mathcal{A}, \mathbf{P})$. If $\zeta_{n} \rightarrow \zeta$ in $D$-distance, this convergence also holds in probability for the usual metric.

For $z$ and $z^{\prime}$ in $\mathbf{C}^{*}$, if $|z| \neq\left|z^{\prime}\right|$, one has $d\left(z, z^{\prime}\right)=2 \pi$; and if $|z|=\left|z^{\prime}\right|$, one has $\left|z^{\prime}-z\right| \leq|z| d\left(z, z^{\prime}\right)$. So, for all $z$ and $z^{\prime}$ in $\mathbf{C}^{*}$,

$$
\left|z^{\prime}-z\right| \wedge 2 \pi \leq(1+|z|) d\left(z, z^{\prime}\right)
$$

This estimate gives

$$
\left|\zeta_{n}-\zeta\right| \wedge 2 \pi \leq(1+|\zeta|) d\left(\zeta, \zeta_{n}\right)
$$

Hence if $d\left(\zeta_{n}, \zeta\right)$ tends to zero in probability, so does $\left|\zeta_{n}-\zeta\right|$.

When $\varepsilon \in[0, \pi]$, we denote by $z \underset{\varepsilon}{\simeq} z^{\prime}$ the relation $d\left(z, z^{\prime}\right) \leq \varepsilon$, which means that $z^{\prime}=z \mathrm{e}^{\mathrm{i} \theta}$ for some $\theta \in[-\varepsilon, \varepsilon]$.

Given $\varepsilon \in] 0, \pi]$, set $m_{\varepsilon}=\lceil 2 \pi / \varepsilon\rceil=\min \{n \in \mathbf{N}: \pi / n \leq \varepsilon / 2\}$. For every $z \in \mathbf{C}^{*}$, the definition

$$
z^{\varepsilon}=|z| \mathrm{e}^{\mathrm{i} 2 k \pi / m_{\varepsilon}} \text { if } z=|z| \mathrm{e}^{\mathrm{i} \theta} \text { with }(2 k-1) \pi / m_{\varepsilon}<\theta \leq(2 k+1) \pi / m_{\varepsilon}
$$

provides an approximation $z^{\varepsilon}$ of $z$ such that $z \underset{\varepsilon / 2}{\simeq} z^{\varepsilon}$; the modulus of $z^{\varepsilon}$ is the same as the modulus of $z$, and the argument of $z^{\varepsilon}$ is the argument of the closest $m_{\varepsilon}$-th root of unity. When $z$ belongs to the set

$$
\Delta_{\varepsilon}=\bigcup_{k=0}^{m_{\varepsilon}-1} \mathbf{R}_{+} \mathrm{e}^{\mathrm{i}(2 k+1) \pi / m_{\varepsilon}}
$$

which consists of $m_{\varepsilon}$ rays separating the roots of unity, the root of unity closest to $z$ is not unique, and an arbitrary choice has been made. The map $z \mapsto z^{\varepsilon}$ is continuous on $\mathbf{C} \backslash \Delta_{\varepsilon}$ for the usual distance, and locally constant on $\mathbf{C} \backslash \Delta_{\varepsilon}$ for the distance $d$.

\subsection{Modifying the increments before a stopping time}

For every stopping time $\tau$ and every complex Brownian motion $C$, we define two new complex Brownian motions $C^{Q, \tau}$ and $C^{Q^{-1}, \tau}$ by

$$
\begin{gathered}
\mathrm{d} C^{Q, \tau}=\left(\mathbf{1}_{[0, \tau]} Q+\mathbf{1}_{] \tau,+\infty[} I_{2}\right) \cdot \mathrm{d} C \\
\mathrm{~d} C^{Q^{-1}, \tau}=\left(\mathbf{1}_{[0, \tau]} Q^{-1}+\mathbf{1}_{] \tau,+\infty[} I_{2}\right) \cdot \mathrm{d} C .
\end{gathered}
$$

Given $s>0$ and $\varepsilon>0$, we introduce the stopping time

$$
\tau_{s, \varepsilon}=\inf \left\{t \geq s: \operatorname{Sol}\left(W, s, t, Z_{s}^{\varepsilon}\right) \underset{\varepsilon / 2}{\simeq} \operatorname{Sol}(Q \cdot W, s, t, \sqrt{s})\right\} .
$$

In this formula, $s$ is a time, but $\sqrt{s}$ (considered as a complex number) is a spatial position; due to Brownian scaling invariance,

$$
\tau_{s, \varepsilon} \quad \text { has the same law as } s \tau_{1, \varepsilon} \text {. }
$$

Note that the definition of $\tau_{s, \varepsilon}$ involves $Z_{s}^{\varepsilon}$ but not $Z_{s}$; since almost surely $Z_{s} \notin \Delta_{\varepsilon}$, a small perturbation of the argument of $Z_{s}$ does not change $Z_{s}^{\varepsilon}, \tau_{s, \varepsilon}$ and $W^{Q, \tau_{s, \varepsilon}}$. Moreover, parallel evolution of

$$
Z=\operatorname{Sol}\left(W, s, \cdot, Z_{s}\right) \text { and } \operatorname{Sol}\left(W, s, \cdot, Z_{s}^{\varepsilon}\right) \quad \text { on }[s,+\infty[
$$

and parallel evolution of

$$
\operatorname{Sol}\left(W, s, \cdot, Z_{s}^{\varepsilon}\right) \text { and } \operatorname{Sol}\left(W^{Q, \tau_{s, \varepsilon}}, s, \cdot \sqrt{s}\right) \text { on the interval }\left[\tau_{s, \varepsilon},+\infty[\right.
$$


show that

$$
Z \underset{\varepsilon / 2}{\simeq} \operatorname{Sol}\left(W, s, \cdot, Z_{s}^{\varepsilon}\right) \underset{\varepsilon / 2}{\simeq} \operatorname{Sol}\left(W^{Q, \tau_{s, \varepsilon}}, s, \cdot, \sqrt{s}\right) \quad \text { on }\left[\tau_{s, \varepsilon},+\infty[\right.
$$

This establishes the next lemma.

For $s>0$ and $\varepsilon>0$, the process $W^{Q, \tau_{s, \varepsilon}}$ is an $\mathcal{F}^{Z}$-Brownian motion. Moreover,

$$
Z \underset{\varepsilon}{\simeq} \operatorname{Sol}\left(W^{Q, \tau_{s, \varepsilon}}, s, \cdot \sqrt{s}\right) \quad \text { on the interval }\left[\tau_{s, \varepsilon},+\infty[.\right.
$$

We will see later that $\tau_{s, \varepsilon}$ is a.s. finite; the scaling property (5) will then show that $\tau_{s, \varepsilon} \rightarrow 0$ in probability when $s \rightarrow 0$. Thus, when $s$ is small enough, knowing the increments after $s$ of the Brownian motion $W^{Q, \tau_{s, \varepsilon}}$ is sufficient to approach $Z_{t}$.

In order to get a Brownian motion $\widehat{W}=\int H \cdot \mathrm{d} W$ on the time interval $[0,1] \mathrm{such}$ that $Z_{1}$ can be recovered from $\left(\widehat{W}_{t}\right)_{0 \leq t \leq 1}$, we shall concatenate pieces of Brownian motions $W^{Q, \tau_{s_{n}, \varepsilon_{n}}}$ for some decreasing sequences $\left(s_{n}\right)_{n \geq 0}$ and $\left(\varepsilon_{n}\right)_{n \geq 0}$ tending to 0.

Assume that, for some $s>0$, we have constructed an approximation of $Z_{s}$ using the Brownian motion $W^{Q, \tau_{r, \delta}}$ for some $\left.s \in\right] 0, r[$. How can we now utilize this approximation and the Brownian motion $W^{Q, \tau_{s, \varepsilon}}$ to approach $Z_{t}$ for $t \geq s$ ? This is the role of the random maps $F_{C, s, t}^{\varepsilon}$ that we now introduce.

\subsection{The random maps $F_{C, s, t}^{\varepsilon}$}

Let $C$ be any $\mathcal{F}^{Z}$-complex Brownian motion. For $s>0, \varepsilon>0$ and $\zeta$ a $\mathbf{C}^{*}$-valued, $\mathcal{F}_{s}^{Z}$-measurable r.v., we define the stopping time

$$
\tau_{C, s, \zeta}^{\varepsilon}=\inf \left\{t \geq s: \operatorname{Sol}\left(Q^{-1} \cdot C, s, t, \zeta^{\varepsilon}\right) \underset{\varepsilon / 2}{\simeq} \operatorname{Sol}(C, s, t, \sqrt{s})\right\}
$$

and for $t \geq s$ we set

$$
F_{C, s, t}^{\varepsilon}(\zeta)=F^{\varepsilon}(C, s, t, \zeta)=\operatorname{Sol}\left(C^{\left.Q^{-1}, \tau_{C, s, \zeta}^{\varepsilon}, s, t, \zeta\right)}\right.
$$

In particular, taking $\zeta$ a constant r.v. $z$, we get an almost surely well defined random map $z \mapsto F_{C, s, t}^{\varepsilon}(z)$ from $\mathbf{C}^{*}$ to $\mathbf{C}^{*}$, and $F_{C, s, t}^{\varepsilon}(\zeta)$ can be identified with the compound $F_{C, s, t}^{\varepsilon} \circ \zeta$.

As a consequence of Lemma 1.1,

$$
F_{C, s, t}^{\varepsilon}(\zeta) \text { is measurable for } \sigma\left(\left(C_{r}-C_{s}\right)_{r \in[s, t]}, \zeta\right) ;
$$

this will be useful later.

When $C=W^{Q, \tau_{s, \varepsilon}}$, the Brownian motions $C$ and $Q \cdot W$ coincide up to time $\tau_{s, \varepsilon}$, so $\tau_{C, s, Z_{s}}^{\varepsilon}=\tau_{s, \varepsilon}$ and $C^{Q^{-1}, \tau_{C, s, Z_{s}}^{\varepsilon}}=W$. Thus

$$
F^{\varepsilon}\left(W^{Q, \tau_{s, \varepsilon}}, s, t, Z_{s}\right)=\operatorname{Sol}\left(W, s, t, Z_{s}\right)=Z_{t}
$$

It will also be useful to know that if $\zeta$ is a complex random variable close to $Z_{s}$, the random variable $F^{\varepsilon}\left(W^{Q, \tau_{s, \varepsilon}}, s, t, \zeta\right)$ is close to $Z_{t}$. We now establish this continuity property of the map $F_{W^{Q, \tau_{s, \varepsilon}}}^{\varepsilon}$.

Fix $\varepsilon>0, s>0$ and $C$ a complex Brownian motion in $\mathcal{F}^{Z}$; let also $\zeta_{0}$ be any complex, $\mathcal{F}_{s}^{Z}$-measurable r.v such that $\mathbf{P}\left[\zeta_{0} \in \Delta_{\varepsilon}\right]=0$. The map $\zeta \mapsto$ $F_{C, s, t}^{\varepsilon}(\zeta)$, defined on the set of all $\mathbf{C}^{*}$-valued, $\mathcal{F}_{s}^{Z}$-measurable random variables, is continuous at $\zeta_{0}$ in the $D$-distance. In other terms, $D\left(F_{C, s, t}^{\varepsilon}(\zeta), F_{C, s, t}^{\varepsilon}\left(\zeta_{0}\right)\right) \rightarrow 0$ when $D\left(\zeta, \zeta_{0}\right) \rightarrow 0$.

On the event $\left[d\left(\zeta, \zeta_{0}\right)<d\left(\zeta_{0}, \Delta_{\varepsilon}\right)\right]$, one has $d\left(\zeta, \zeta_{0}\right)<2 \pi$, whence $|\zeta|=\left|\zeta_{0}\right|$; moreover, $\zeta$ and $\zeta_{0}$ are in the same connected component of $\Delta_{\varepsilon}^{\mathrm{c}}$, so $\zeta_{0}^{\varepsilon}=\zeta^{\varepsilon}$. This implies $\tau_{C, s, \zeta}^{\varepsilon}=\tau_{C, s, \zeta_{0}}^{\varepsilon}$, and consequently also $C^{Q, \tau_{C, s, \zeta}^{\varepsilon}}=C^{Q, \tau_{C, s, \zeta_{0}}^{\varepsilon}}$; by Corollary $1.2, d\left(F_{C, s, t}^{\varepsilon}(\zeta), F_{C, s, t}^{\varepsilon}\left(\zeta_{0}\right)\right) \leq d\left(\zeta, \zeta_{0}\right)$. 
by $2 \pi$.

On the complementary event $\left[d\left(\zeta, \zeta_{0}\right) \geq d\left(\zeta_{0}, \Delta_{\varepsilon}\right)\right]$, we simply majorize $d\left(F_{C, s, t}^{\varepsilon}(\zeta), F_{C, s, t}^{\varepsilon}\left(\zeta_{0}\right)\right)$

All in all,

$$
d\left(F_{C, s, t}^{\varepsilon}(\zeta), F_{C, s, t}^{\varepsilon}\left(\zeta_{0}\right)\right) \leq d\left(\zeta, \zeta_{0}\right)+2 \pi \mathbf{1}_{d\left(\zeta, \zeta_{0}\right) \geq d\left(\zeta_{0}, \Delta_{\varepsilon}\right)} ;
$$

and taking expectations gives

$$
D\left(F_{C, s, t}^{\varepsilon}(\zeta), F_{C, s, t}^{\varepsilon}\left(\zeta_{0}\right)\right) \leq \mathbf{E}\left[d\left(\zeta, \zeta_{0}\right)\right]+2 \pi \mathbf{P}\left[d\left(\zeta, \zeta_{0}\right) \geq d\left(\zeta_{0}, \Delta_{\varepsilon}\right)\right] .
$$

By hypothesis, the r.v. $d\left(\zeta_{0}, \Delta_{\varepsilon}\right)$ is a.s. $>0$; so when $d\left(\zeta, \zeta_{0}\right)$ tends to zero in probability, the right-hand side tends to zero.

\section{Construction of $\widehat{W}=\int H \cdot \mathrm{d} W$ on the time inter- val $[0,1]$}

The construction will rest on the continuity properties of the maps $F_{C, s, t}^{\varepsilon}$ (Lemma 1.4) and on the next statement, which will be admitted in subsection 2.1 , and proved in subsection 2.2 as a consequence of the convergence $P\left[\tau_{s, \varepsilon} \leq t\right]=P\left[\tau_{1, \varepsilon} \leq t / s\right] \rightarrow 1$ as $s \rightarrow 0$.

For all $t \geq s>0$ and $\varepsilon>0$,

$$
F_{W^{Q, \tau_{s, \varepsilon}, s, t}}^{\varepsilon}(\sqrt{s})=\operatorname{Sol}\left(W^{Q, \tau_{s, \varepsilon}}, s, t, \sqrt{s}\right) .
$$

Moreover, given $t>0$ and $\varepsilon>0, D\left(Z_{t}, F_{W^{Q, \tau_{s, \varepsilon}, s, t}}^{\varepsilon}(\sqrt{s})\right)<2 \varepsilon$ provided $s$ is small enough.

\subsection{The construction}

First, we recursively define two decreasing sequences $\left(s_{n}\right)_{n \geq 0}$ and $\left(\varepsilon_{n}\right)_{n \geq 0}$ such that for every $n \geq 1, D\left(Z_{1}, G_{n}\left(\sqrt{s_{n}}\right)\right) \leq 1 / n$, with

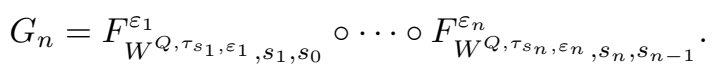

The construction begins with $s_{0}=1$ and $\varepsilon_{0}=1$.

Assume that, for some $n \geq 1, s_{0}>\ldots>s_{n-1}>0$ and $\varepsilon_{0}>\ldots>\varepsilon_{n-1}>0$ have already been constructed. Property (7) says that $F_{W^{Q, \tau_{s, \varepsilon}, s, t}}^{\varepsilon}\left(Z_{s}\right)=Z_{t}$ for $t \geq s$, so $G_{n-1}\left(Z_{s_{n-1}}\right)=Z_{s_{0}}=Z_{1}$; and Lemma 1.4 says that the map $\zeta \mapsto G_{n-1}(\zeta)$ is $D$-continuous at $Z_{s_{n-1}}$. This provides some $\left.\varepsilon_{n} \in\right] 0, \varepsilon_{n-1}[$ such that, for each $\zeta$ measurable in $\mathcal{F}_{s_{n-1}}^{Z}$,

$$
D\left(Z_{s_{n-1}}, \zeta\right) \leq 2 \varepsilon_{n} \quad \Longrightarrow \quad D\left(Z_{1}, G_{n-1}(\zeta)\right) \leq 1 / n .
$$

Then Lemma 2 (provisionally admitted) provides some $\left.s_{n} \in\right] 0, s_{n-1} / 2[$ such that

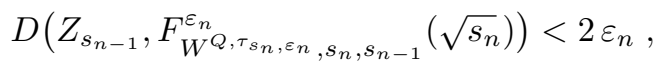

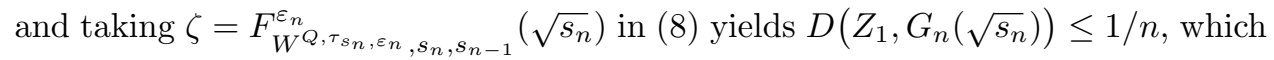
completes the recursion.

Let now $H$ be the process defined on the interval $] 0,1]$ by

$$
\left.\left.H_{s}=Q \mathbf{1}_{\left[s \leq \tau_{s_{n}, \varepsilon_{n}}\right]}+I_{2} \mathbf{1}_{\left[s>\tau_{s_{n}, \varepsilon_{n}}\right]} \quad \text { on the interval }\right] s_{n}, s_{n-1}\right] .
$$

As $H$ takes its values in $\left\{I_{2}, Q\right\}$, the process $\widehat{W}=\int H \cdot \mathrm{d} W$ is a complex Brownian motion. By construction, $\widehat{W}$ has the same increments as $W^{Q, \tau_{s_{n}, \varepsilon_{n}}}$ on the interval ]$\left.s_{n}, s_{n-1}\right]$. By Lemma 1 , we only have to show that the r.v. $Z_{1}$ can be recovered from the observation of $\widehat{W}$ on $[0,1]$. 
Define a family $\left(\zeta_{n, k}\right)_{1 \leq k \leq n}$ of $\mathbf{C}$-valued random variables by $\zeta_{n, n}=\sqrt{s_{n}}$ and, for $k$ decreasing from $n$ to 1 ,

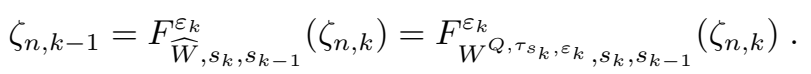

Property (6) shows by recursion that $\zeta_{n, k}$ is $\mathcal{F}_{s_{k}}$-measurable; in particular $\zeta_{n, 1}$ is $\mathcal{F}_{1}^{W}$-measurable. By definition of $G_{n}$, one has $\zeta_{n, 1}=G_{n}\left(\sqrt{s_{n}}\right)$, wherefrom $D\left(Z_{1}, \zeta_{n, 1}\right) \leq 1 / n$. By Lemma $1.2, \zeta_{n, 1} \rightarrow Z_{1}$ in probability, and $Z_{1}$ too is $\mathcal{F}_{1}^{W_{-}}$ measurable.

\subsection{Proof of Lemma 2}

The last missing step in the proof is Lemma 2, to be proved now. A key point in this proof is the following coupling property.

The orthogonal matrix

$$
Q=\left(\begin{array}{cc}
a & -\sigma b \\
b & \sigma a
\end{array}\right)
$$

is still fixed, with $a$ and $b$ in $\mathbf{R}, a^{2}+b^{2}=1$ and $\sigma \in\{-1,1\}$. Assume that $(a, b) \neq(1,0)$. Then, given $\varepsilon>0$, the stopping time

$$
\tau_{1, \varepsilon}=\inf \left\{t \geq 1: \operatorname{Sol}\left(W, 1, t, Z_{1}^{\varepsilon}\right) \underset{\varepsilon / 2}{\simeq} \operatorname{Sol}(Q \cdot W, 1, t, 1)\right\}
$$

is almost surely finite.

We stress the fact that the conclusion turns out to be false if one takes $(a, b)=$ $(1,0)$. This case will be discussed in subsection 2.4 .

Proof of Lemma 2, assuming Lemma 2.2. Fix $t \geq s>0$, and let $C$ be any complex $\mathcal{F}^{Z}$-Brownian motion. Since the stopping time $\tau_{C, s, \zeta}^{\varepsilon}$ defined in subsection 1.4 is

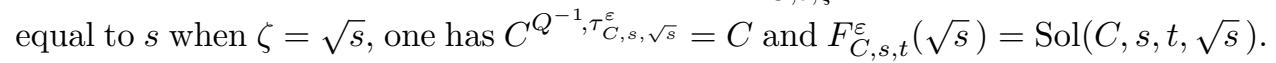
Taking in particular $C=W^{Q, \tau_{s, \varepsilon}}$ yields the first part of Lemma 2.

By Lemma $1.3, Z_{t} \underset{\varepsilon}{\simeq} \operatorname{Sol}\left(W^{Q, \tau_{s, \varepsilon}}, s, t, \sqrt{s}\right)$ on the event $\left[\tau_{s, \varepsilon} \leq t\right]$, so

$$
\begin{aligned}
D\left(Z_{t}, \operatorname{Sol}\left(W^{Q, \tau_{s, \varepsilon}}, s, t, \sqrt{s}\right)\right) & \leq \varepsilon P\left[\tau_{s, \varepsilon} \leq t\right]+2 \pi P\left[\tau_{s, \varepsilon}>t\right] \\
& \leq \varepsilon+2 \pi P\left[\tau_{s, \varepsilon}>t\right] .
\end{aligned}
$$

Now, by Lemma 2.2 and by the scaling property (5),

$$
P\left[\tau_{s, \varepsilon}>t\right]=P\left[\tau_{1, \varepsilon}>t / s\right] \rightarrow 0 \quad \text { as } \quad s \rightarrow 0 ;
$$

hence $\limsup _{s \rightarrow 0} D\left(Z_{t}, \operatorname{Sol}\left(W^{Q, \tau_{s, \varepsilon}}, s, t, \sqrt{s}\right)\right) \leq \varepsilon$.

\subsection{Proof of Lemma 2.2}

By parallel evolution, for every $t \geq 1, \operatorname{Sol}\left(W, 1, t, Z_{1}^{\varepsilon}\right)=\left(Z_{1}^{\varepsilon} / Z_{1}\right) \times Z_{t}$. Set $Z_{t}^{\prime}=$ $\operatorname{Sol}\left(Q \cdot W, 1, t, Z_{1} / Z_{1}^{\varepsilon}\right)=\left(Z_{1} / Z_{1}^{\varepsilon}\right) \times \operatorname{Sol}(Q \cdot W, 1, t, 1)$. Then

$$
\left.\tau_{1, \varepsilon}=\inf \left\{t \geq 1: Z_{t} \underset{\varepsilon / 2}{\simeq} Z_{t}^{\prime}\right)\right\}
$$

For $t \geq 1$, set $Z_{t}=R_{t} \mathrm{e}^{\mathrm{i} \Theta_{t}}$ and $Z_{t}^{\prime}=R_{t}^{\prime} \mathrm{e}^{\mathrm{i} \Theta_{t}^{\prime}}$, where $\Theta$ and $\Theta^{\prime}$ are continuous and $\mathcal{F}^{\bar{Z}}$-adapted determinations of the arguments of $Z$ and $Z^{\prime}$ on the time-interval $[1,+\infty[$, and put

$$
L_{t}=\ln R_{t}-\ln R_{t}^{\prime}, \quad M_{t}=\Theta_{t}-\Theta_{t}^{\prime} .
$$

With this notation, $\tau_{1, \varepsilon}$ becomes

$$
\tau_{1, \varepsilon}=\inf \left\{t \geq 1: L_{t}=0 \text { and } M_{t} \in[-\varepsilon / 2, \varepsilon / 2]+2 \pi \mathbf{Z}\right\} .
$$

Set $c=a+\mathrm{i} b$. By assumption, $c$ has modulus 1 and $c \neq 1$. Two cases will be distinguished according to the value of $\sigma$. 
1. Case where $\sigma=1$. If $\sigma=1$, then $Q \cdot W=c W$, and stochastic calculus yields

$$
\mathrm{d}\left(L_{t}+\mathrm{i} M_{t}\right)=\frac{\mathrm{d} Z_{t}}{Z_{t}}-\frac{\mathrm{d} Z_{t}^{\prime}}{Z_{t}^{\prime}}=\left(\frac{1}{R_{t}}-\frac{c}{R_{t}^{\prime}}\right) \mathrm{d} W_{t} ;
$$

so $L+\mathrm{i} M$ is a conformal local martingale. Now, $\left|\left(1 / R_{t}\right)-\left(c / R_{t}^{\prime}\right)\right|$ can be minorated by the imaginary part $|b| / R_{t}^{\prime}$; or, if $b=0$ and $c=-1$, by $1 / R_{t}^{\prime}$. This minoration entails that

$$
\langle L\rangle_{t}=\langle M\rangle_{t}=\int_{1}^{t}\left|\frac{1}{R_{s}}-\frac{c}{R_{s}^{\prime}}\right|^{2} \mathrm{~d} s \rightarrow+\infty \quad \text { as } \quad t \rightarrow+\infty,
$$

since the time spent by $R^{\prime}$ below any level is infinite. Consequently, $L+\mathrm{i} M$ is a timechanged complex Brownian motion, and it almost surely visits the set $\mathrm{i}([-\varepsilon / 2, \varepsilon / 2]+$ $2 \pi \mathbf{Z})$; so $\tau_{1, \varepsilon}$ is finite.

2. Case where $\sigma=-1$. If $\sigma=-1$, then $Q \cdot W=c \bar{W}$, and one has

$$
\mathrm{d}\left(L_{t}+\mathrm{i} M_{t}\right)=\frac{\mathrm{d} Z_{t}}{Z_{t}}-\frac{\mathrm{d} Z_{t}^{\prime}}{Z_{t}^{\prime}}=\frac{\mathrm{d} W_{t}}{R_{t}}-\frac{c \mathrm{~d} \bar{W}_{t}}{R_{t}^{\prime}} .
$$

We can define a real local martingale $\left(N_{t}\right)_{t \geq 1}$ by $N_{1}=0$ and

$$
\operatorname{id}\left(N_{t}-M_{t}\right)=\frac{1}{R_{t}^{\prime}}\left(c \mathrm{~d} \bar{W}_{t}-\bar{c} \mathrm{~d} W_{t}\right)
$$

Observe that

$$
\mathrm{d}\left(L_{t}+\mathrm{i} N_{t}\right)=\left(\frac{1}{R_{t}}-\frac{\bar{c}}{R_{t}^{\prime}}\right) \mathrm{d} W_{t}=\frac{R_{t}^{\prime}-\bar{c} R_{t}}{R_{t} R_{t}^{\prime}} \mathrm{d} W_{t},
$$

so $L+\mathrm{i} N$ is a conformal local martingale and

$$
\frac{c \mathrm{~d} \bar{W}_{t}}{R_{t}^{\prime}}=\frac{c R_{t}}{R_{t}^{\prime}-c R_{t}} \mathrm{~d}\left(L_{t}-\mathrm{i} N_{t}\right) .
$$

But using $c=a+i b, c \bar{c}=1$ and $R_{t} / R_{t}^{\prime}=\mathrm{e}^{L_{t}}$, one gets

$$
\frac{c R_{t}}{R_{t}^{\prime}-c R_{t}}=\frac{c R_{t}\left(R_{t}^{\prime}-\bar{c} R_{t}\right)}{R_{t}^{\prime 2}-2 a R_{t} R_{t}^{\prime}+R_{t}^{2}}=\frac{c-\mathrm{e}^{L_{t}}}{\mathrm{e}^{-L_{t}}-2 a+\mathrm{e}^{L_{t}}}=\frac{c-\mathrm{e}^{L_{t}}}{2\left(\cosh \left(L_{t}\right)-a\right)} .
$$

Hence

$$
\mathrm{d}\left(N_{t}-M_{t}\right)=2 \Im\left(\frac{c \mathrm{~d} \bar{W}_{t}}{R_{t}^{\prime}}\right)=\frac{b \mathrm{~d} L_{t}-a \mathrm{~d} N_{t}+\mathrm{e}^{L_{t}} \mathrm{~d} N_{t}}{\cosh \left(L_{t}\right)-a},
$$

or equivalently,

$$
\mathrm{d} M_{t}=\frac{-b \mathrm{~d} L_{t}-\sinh \left(L_{t}\right) \mathrm{d} N_{t}}{\cosh \left(L_{t}\right)-a} .
$$

Moreover, $\langle L, N\rangle_{t}=0$ because $L+\mathrm{i} N$ is conformal, and

$$
\langle L\rangle_{t}=\langle N\rangle_{t}=\int_{1}^{t}\left|\frac{1}{R_{s}}-\frac{\bar{c}}{R_{s}^{\prime}}\right|^{2} \mathrm{~d} s .
$$

As in the first case, the continuous, strictly increasing process $\langle L\rangle=\langle N\rangle$ starts from 0 at time 1 and goes to $+\infty$. Hence, on the time-interval $[1,+\infty[$, the local martingale $(L, N)$ is a time-changed two-dimensional Brownian motion.

Call $\left(\alpha_{s}\right)_{s \geq 0}$ the inverse of the process $\langle L\rangle=\langle N\rangle$, and set

$$
\lambda_{s}=L_{\alpha_{s}}, \mu_{s}=M_{\alpha_{s}} \text { and } \nu_{s}=N_{\alpha_{s}} .
$$


Then the processes $\lambda$ and $\nu$ are independent one-dimensional Brownian motions and (9) becomes

$$
\mathrm{d} \mu_{s}=-\frac{b}{\cosh \left(\lambda_{s}\right)-a} \mathrm{~d} \lambda_{s}-\frac{\sinh \left(\lambda_{s}\right)}{\cosh \left(\lambda_{s}\right)-a} \mathrm{~d} \nu_{s} .
$$

To prove that $\tau_{1, \varepsilon}$ is (almost surely) finite, we have to show that the process $(\lambda, \mu)$ almost surely visits the set $\{0\} \times([-\varepsilon / 2, \varepsilon / 2]+2 \pi \mathbf{Z})$.

Changing time again, call $\varrho$ the right-continuous inverse of the local time of $\lambda$ at 0 . Observing that $\lambda_{\varrho_{\ell}}=0$, it now suffices to verify that the process $\left(\mu_{\varrho_{\ell}}\right)_{\ell \geq 0}$ visits $[-\varepsilon / 2, \varepsilon / 2]+2 \pi \mathbf{Z}$.

For every $\ell \geq 0$, set

$$
S_{\ell}=\mu_{0}-\mu_{\varrho_{\ell}}=\int_{0}^{\varrho_{\ell}} \frac{b}{\cosh \left(\lambda_{s}\right)-a} \mathrm{~d} \lambda_{s}+\int_{0}^{\varrho_{\ell}} \frac{\sinh \left(\lambda_{s}\right)}{\cosh \left(\lambda_{s}\right)-a} \mathrm{~d} \nu_{s} ;
$$

The Markov property of Brownian motion and the independence of $\lambda$ and $\nu$ imply that, conditionally on $\lambda, S$ is a Gaussian process with independent increments, and the variance of $S_{\ell}$ is

$$
\int_{0}^{\varrho_{\ell}}\left(\frac{\sinh \left(\lambda_{s}\right)}{\cosh \left(\lambda_{s}\right)-a}\right)^{2} \mathrm{~d} s
$$

This variance tends to infinity with $\ell$ because the time spent by $\lambda$ outside $[-1,1]$ is infinite. Hence, the conclusion follows from the next lemma.

Let $\left(S_{t}\right)_{t>0}$ be a Gaussian process with independent increments, such that $\operatorname{Var}\left(S_{t}\right) \rightarrow+\infty$ as $t \rightarrow+\infty$. Then almost surely, for every interval $I$ with positive length, $\left(S_{t}\right)_{t>0}$ visits $I+2 \pi \mathbf{Z}$.

First, notice that if $G$ is a Gaussian random variable with law $\mathcal{N}(m, v)$, then the law of $G-m$ is $\mathcal{N}(0, v)$; hence, calling $g_{v}$ the density of $\mathcal{N}(0, v)$, one has, for every interval $I$ with length $\leq 2 \pi$,

$$
P[G \in I+2 \pi \mathbf{Z}]=\sum_{k \in \mathbf{Z}} P[G \in I+2 \pi k]=\int_{I-m}\left(\sum_{k \in \mathbf{Z}} g_{v}(x-k 2 \pi)\right) \mathrm{d} x .
$$

Now, the Poisson summation formula yields, for every $x \in \mathbf{R}$,

$$
\sum_{k \in \mathbf{Z}} g_{v}(x-k 2 \pi)=\frac{1}{2 \pi} \sum_{n \in \mathbf{Z}} \mathrm{e}^{-v n^{2} / 2} \mathrm{e}^{\mathrm{i} n x} .
$$

If $v \geq 2$,

$$
2 \pi \sum_{k \in \mathbf{Z}} g_{v}(x-k 2 \pi) \geq 1-2 \sum_{n \geq 1} \mathrm{e}^{-v n^{2} / 2} \geq 1-2 \sum_{n \geq 1} \mathrm{e}^{-n^{2}} \geq \frac{1}{5},
$$

hence for every interval $I$ with length $\leq 2 \pi$,

$$
P[G \in I+2 \pi \mathbf{Z}] \geq \frac{1}{10 \pi}|I| .
$$

For every $t \geq 0$, set $V(t)=\operatorname{Var}\left(S_{t}\right)$. Since $V(t) \rightarrow+\infty$ as $t \rightarrow+\infty$, one can recursively construct an increasing sequence $\left(t_{n}\right)_{n \geq 1}$ such that $V\left(t_{1}\right) \geq 2$ and $V\left(t_{n}\right) \geq V\left(t_{n-1}\right)+2$ for every $n \geq 2$. The random variables $S_{t_{1}}$ and $\left(S_{t_{n}}-S_{t_{n-1}}\right)_{n \geq 2}$ are independent, Gaussian with variance $\geq 2$, so for every $n \geq 1$,

$$
\mathbf{P}\left[S_{t_{n}} \in I+2 \pi \mathbf{Z} \mid \sigma\left(S_{t_{1}}, \ldots, S_{t_{n-1}}\right)\right] \geq \frac{|I|}{10 \pi}
$$

and by recursion,

$$
\mathbf{P}\left[\forall k \in[1 \ldots n], S_{t_{k}} \notin I+2 \pi \mathbf{Z}\right] \leq\left(1-\frac{|I|}{10 \pi}\right)^{n}
$$

Hence

$$
\mathbf{P}\left[\forall k \geq 1, S_{t_{k}} \notin I+2 \pi \mathbf{Z}\right]=0 .
$$

This provides the results for a given interval $I$. Letting the bounds of the interval vary in $\mathbf{Q}$ yields the result. 


\subsection{The case when $(a, b)=(1,0)$}

Assume now that $(a, b)=(1,0)$. First, we explain why the conclusion of Lemma 2.2 does not hold any longer.

The case when $\sigma=1$ is not interesting in view of Theorem since it corresponds to the choice $Q=I_{2}$. In that case, both processes $\operatorname{Sol}\left(W, 1, t, Z_{1}^{\varepsilon}\right)$ and $\operatorname{Sol}(Q \cdot W, 1, t, 1)$ evolve parallelly, and by the remark after Corollary 1.2, the stopping time $\tau_{1, \varepsilon}$ is finite only on the null event $\left[Z_{1} \underset{\varepsilon / 2}{\simeq} 1\right]$.

The case when $\sigma=-1$ is much more interesting since it corresponds to the complementability of $U$. In that case, $Q$ is diagonal with diagonal $(1,-1)$, and $Q \cdot W=\bar{W}=U-\mathrm{i} V$. Thus the processes $R$ and $R^{\prime}$, introduced in the proof of Lemma 2.2, are solutions of stochastic differential equations governed by the same Brownian motion $U$, namely,

$$
\mathrm{d} R_{s}=\mathrm{d} U_{s}+\frac{\mathrm{d} s}{2 R_{s}} \quad \text { and } \quad \mathrm{d} R_{s}^{\prime}=\mathrm{d} U_{s}+\frac{\mathrm{d} s}{2 R_{s}^{\prime}}
$$

with initial conditions $R_{1}=\left|Z_{1}\right|$ and $R_{1}^{\prime}=1$. Almost surely, $\left|Z_{1}\right| \neq 1$; hence, by noticing that the quantity

$$
\ln \left|R_{t}-R_{t}^{\prime}\right|=\ln \left|R_{1}-R_{1}^{\prime}\right|-\int_{1}^{t} \frac{\mathrm{d} s}{2 R_{s} R_{s}^{\prime}}
$$

does not explode in finite time, or by applying classical results on flows of solutions of SDEs, one sees that the processes $R$ and $R^{\prime}$ never meet, so the stopping time $\tau_{1, \varepsilon}$ is infinite.

Still, the proof of Theorem can be adapted to the case when $(a, b)=(1,0)$ and $\sigma=-1$; it even becomes simpler, and, in fact, reduces to the proof given in [2]. Here are the changes to be made in the preceding proof.

First, the stopping times $\tau_{s, \varepsilon}$ must be replaced with the stopping times

$$
\tau_{s}=\inf \left\{t \geq s: \operatorname{Sol}\left(W, s, t, Z_{s}\right)=\operatorname{Sol}\left(Q \cdot W, s, t, R_{s}\right)\right\} .
$$

Note the modifications in the definition:

- the $\varepsilon / 2$-almost equality becomes a true equality;

- the initial position $Z_{s}^{\varepsilon}$ in $\operatorname{Sol}\left(W, s, t, Z_{s}^{\varepsilon}\right)$ is replaced by $Z_{s}$;

- the initial position $\sqrt{s}$ in $\operatorname{Sol}(Q \cdot W, s, t, \sqrt{s})$ is replaced by $R_{s}$.

Choosing $R_{s}$ as the initial position will not cause any difficulty since for every previsible process $H$ with values in $\left\{I_{2}, Q\right\}$, the real part of the Brownian motion $\widehat{W}=\int H \cdot \mathrm{d} W$ is $U$, so the process $R$ is adapted to $\mathcal{F}^{\widehat{W}}$.

Almost sure finiteness is much more simply proved for $\tau_{1}$ than for $\tau_{1, \varepsilon}$. Indeed, setting $Z_{t}=\operatorname{Sol}\left(W, 1, t, Z_{1}\right)=R_{t} \mathrm{e}^{\mathrm{i} \Theta_{t}}$ for $t \geq 1$ as before, one gets $\operatorname{Sol}(Q$. $\left.W, 1, t, R_{1}\right)=R_{t} \mathrm{e}^{\mathrm{i}\left(\Theta_{0}-\Theta_{t}\right)}$, so

$$
\tau_{1}=\inf \left\{t \geq 1: 2 \Theta_{t} \in \Theta_{0}+2 \pi \mathbf{Z}\right\}
$$

As $\Theta$ is a time-changed Brownian motion, $\tau_{1}$ is almost surely finite.

Then we only need to choose any decreasing sequence $\left(s_{n}\right)_{n \geq 0}$ of positive real numbers such that $s_{n} / s_{n-1} \rightarrow 0$ as $n \rightarrow \infty$ (this ensures that $P\left[\tau_{s_{n}} \leq s_{n-1}\right]=$ $\left.P\left[\tau_{1} \leq s_{n-1} / s_{n}\right] \rightarrow 1\right)$, and to define the Brownian motion $\widehat{W}=\int H \cdot \mathrm{d} W$ on the interval $[0,1]$ as before. In the construction proposed in Subsection 2.1, the starting value $\zeta_{n, n}$ of the sequence $\left(\zeta_{n, k}\right)_{k \in\{1, \ldots, n\}}$ also has to be modified; a possible choice is $\zeta_{n, n}=R_{s_{n}}$. 


\section{References}

[1] Brossard J., Émery M. and Leuridan C. (2009) Maximal Brownian motions. Annales de l'Institut Henri Poincaré Probab. Statist. 45-3, 876-886.

[2] Brossard J. and Leuridan C. (2008) Filtrations browniennes et compléments indépendants. Séminaire de Probabilités XLI, Lecture Notes in Mathematics 1934, 265-278, Springer.

[3] Dubins L., Feldman J., Smorodinsky M. and Tsirelson B. (1996) Decreasing sequences of $\sigma$-fields and a measure change for Brownian motion Annals of Probability 24-2, 882-904.

[4] Émery M. (2005) On certain almost Brownian filtrations. Annales de l'Institut Henri Poincaré Probab. Statist. 41-3, 285-305.

[5] Malric M. (2001) Filtrations quotients de la filtration brownienne. Séminaire de Probabilités XXXV, Lecture Notes in Mathematics 1755, 260-264, Springer.

[6] Stroock D. and Yor M. (1980) On extremal solutions of martingale problems. Annales scientifiques de l'École Normale Supérieure 13-1, 95-164.

[7] Stroock D.W. and Yor M. (1981) Some remarkable martingales. Séminaire de Probabilités XV, Lecture Notes in Mathematics 850, 590-603, Springer.

[8] Tsirelson B. (1997) Triple points: from non-Brownian filtrations to harmonic measures. Geometric and Functional Analysis 7-6, 1096-1142.

Jean Brossard

Institut Fourier, Université Joseph Fourier et CNRS

BP 74, 38402 Saint-Martin-d'Hères Cedex, France

Michel Émery

IRMA, Université Unique de Strasbourg et CNRS

7 rue René Descartes, 67084 Strasbourg Cedex, France

Christophe Leuridan

Institut Fourier, Université Joseph Fourier et CNRS

BP 74, 38402 Saint-Martin-d'Hères Cedex, France 